\title{
Low-field accelerator structure couplers and design techniques
}

\author{
Christopher Nantista, Sami Tantawi, and Valery Dolgashev \\ Stanford Linear Accelerator Center (SLAC), 2575 Sand Hill Road, Menlo Park, California 94025, USA
}

(Received 4 February 2004; published 14 July 2004)

\begin{abstract}
Recent experience with $X$-band accelerator structure development has shown the rf input coupler to be the region most prone to rf breakdown and degradation, effectively limiting the operating gradient. A major factor in this appears to be high magnetic fields at the sharp edges of the coupling irises. As a first response to this problem, couplers with rounded and thickened iris horns have been employed and successfully tested at high power. To further reduce fields for higher power flow, conceptually new coupler designs have been developed, in which power is coupled through the broad wall of the feed waveguide, rather than through terminating irises. A "mode-launcher" coupler, which launches the $\mathrm{TM}_{01}$ mode in circular waveguide before coupling through a matching cell into the main structure, has been tested with great success. With peak surface fields below those in the body of the structure, this coupler represented a breakthrough in the Next Linear Collider structure program. The design of this coupler and of variations which use beam line space more efficiently are described here. The latter include a coupler in which power passes directly through an iris in the broad wall of the rectangular waveguide into a matching cell, also successfully implemented, and a variation which makes the waveguide itself an accelerating cell. We also discuss in some detail a couple of techniques for matching such couplers to traveling-wave structures using a field solver. The first exploits the cell number independence of a traveling-wave match, and the second optimizes using the fields of an internally driven structure.
\end{abstract}

DOI: 10.1103/PhysRevSTAB.7.072001

PACS numbers: 29.17.+w, 84.40.Az

\section{INTRODUCTION}

Radio frequency power typically enters and leaves an accelerator structure by magnetic coupling through a pair of iris apertures in thin-walled interfaces between the ends or narrow walls of rectangular waveguides and a coupling cell [1]. In the development of high-gradient structures for future linear colliders, much attention has been demanded by the limitations imposed by rf breakdown and surface damage [2,3]. Although high-field regions such as iris tips and slots throughout these structures are vulnerable to breakdown, couplers have been seen to represent a sort of bottleneck. In the past few years, this has led to the exploration of ways to reduce both the magnetic and electric surface fields in structure couplers. We here present and discuss some solutions to the coupler problem.

Structures developed at SLAC as part of the Next Linear Collider (NLC) program operate at $11.424 \mathrm{GHz}$ and have been tested with peak power levels up to $100 \mathrm{MW}$ and surface gradients up to $200 \mathrm{MV} / \mathrm{m}$. Postprocessing autopsies of test structures have revealed gross deterioration of the "horns" or ridges of waveguide to coupler cell irises. This confirmed a connection between processing damage and relatively large surface currents [4]. The problem was most obvious in structures where the horn edges had been made sharp $(\sim 0.08 \mathrm{~mm}$ radii). The most straightforward remedy to this problem is to round the iris edges with increased radius. Figure 1 shows a coupler geometry of the type which performed poorly and a modified coupler with a $3 \mathrm{~mm}$ thick, full- radiused waveguide iris. This redesign reduced the peak surface magnetic field by $\sim 50 \%$ and the pulsed heating by a factor of 4 . The wall of this new "fat-lipped" coupler cell was given a racetrack shape to compensate for the larger quadrupole distortion of the fields, as suggested by [5]. Five couplers of this type were incorporated in $X$-band accelerating structures, showing improved performance [6].

Further reduction of coupler fields requires more than a dimensional change in the interface between feeding waveguides and the coupler cell. In Sec. II we introduce a more radical change, in which power is coupled to the structure axially, after conversion to the appropriate circular waveguide mode. Modification of this coupler design to make more efficient use of beam line space leads to a very simple geometry, in which power is coupled out through the broad wall of a rectangular feed waveguide, rather than through an inductive cross-sectional iris. With further modification, the space intercepted by the waveguide itself can be reclaimed for optimal acceleration, without unacceptable magnetic field enhancement.
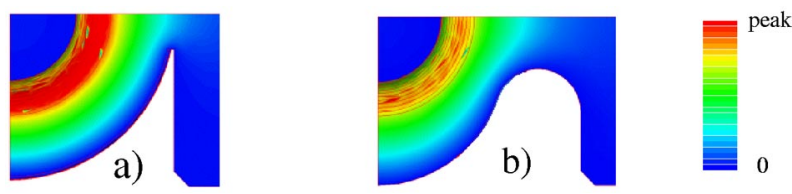

FIG. 1. (Color) Quarter cross section of (a) thin-irised coupler and (b) fat-lipped coupler with reduced surface magnetic field and "racetrack" cell shape. 


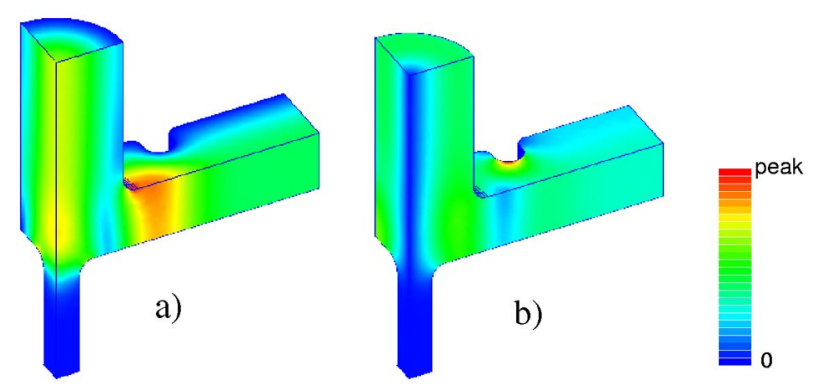

FIG. 2. (Color) Quarter geometry of $\mathrm{TM}_{01}$ mode launcher with (a) electric field and (b) magnetic field plots. Red indicates highest field and blue lowest.

Section III describes methods of designing matching cells for these mode-converter couplers, given the geometry of the nearby structure cells. Our conclusions can be found in Sec. IV.

\section{MODE-CONVERTER COUPLERS}

\section{A. The mode-launcher coupler}

Greater reduction of coupler electric and magnetic fields requires a qualitative change of design. The modelauncher coupler was adapted from a setup designed to test and study single cells of various geometries and materials. To test the performance of these cells without being limited by coupler field enhancement, we had decided to couple on axis from a circular $\mathrm{TM}_{01}$ mode waveguide. This requires a $\mathrm{TM}_{01}$ launcher. Between two such launchers could be inserted a simple, symmetric, traveling-wave structure consisting of the test cell sandwiched between two lower-field matching cells.

The launcher, or mode converter, designed for this consists of a WR90 waveguide, fed symmetrically from both ends, opening through its broad wall into a perpendicular 0.900 in. diameter circular waveguide. Matching the diameter to the rectangular guide width keeps the fields low while avoiding unnecessary overmoding. The only other propagating mode is the fundamental $\mathrm{TE}_{11}$ mode. With symmetric feeding it is excluded by symmetry, and even with imperfect feeding symmetry it is poorly coupled compared to the $\mathrm{TM}_{01}$ mode, due to the field orientation. A simple matching element completes this launcher or mode converter. This can be a ridge, or iris, in the circular waveguide or, as shown in Fig. 2, a set of matching bumps (or posts) in the rectangular waveguide.

The edge of the junction, where the walls of the rectangular and circular guides meet, is rounded to minimize electric field enhancement. For the singe-cell tests, a smaller hole, opposite the circular waveguide, opens into a cutoff viewport. In adapting this mode converter into a coupler for an actual beam line accelerator structure, this viewport becomes the beam pipe.

Figure 2 shows the geometry of the mode launcher with electric and magnetic field plots from HFSS [7]. For a power flow of $50 \mathrm{MW}$, the peak surface electric field is $35 \mathrm{MV} / \mathrm{m}$ and the peak magnetic field is $100 \mathrm{kA} / \mathrm{m}$. The measured match, quite broad relative to typical structure bandwidths, is shown in Fig. 3, along with a transmission measurement.

Use of this universal mode converter in any given input or output coupler requires design of a customized cell to match the circular waveguide into the disk-loaded structure. This cell has fields higher than in the mode converter itself, but can typically be designed to have surface fields below those in the main structure cells, so that the coupler is no longer a weak link. One advantage of the mode-converter coupler is that the structure-specific design work is reduced to a two-dimensional, on-axis coupling problem. Techniques for design of matching cells are treated in Sec. III. Nine mode-launcher couplers have been used in the high-gradient structure program at SLAC. Figure 4 shows a model of such a coupler.

\section{B. The waveguide coupler}

An undesirable aspect of the mode-launcher coupler, where beam line real estate is valuable, is that very little
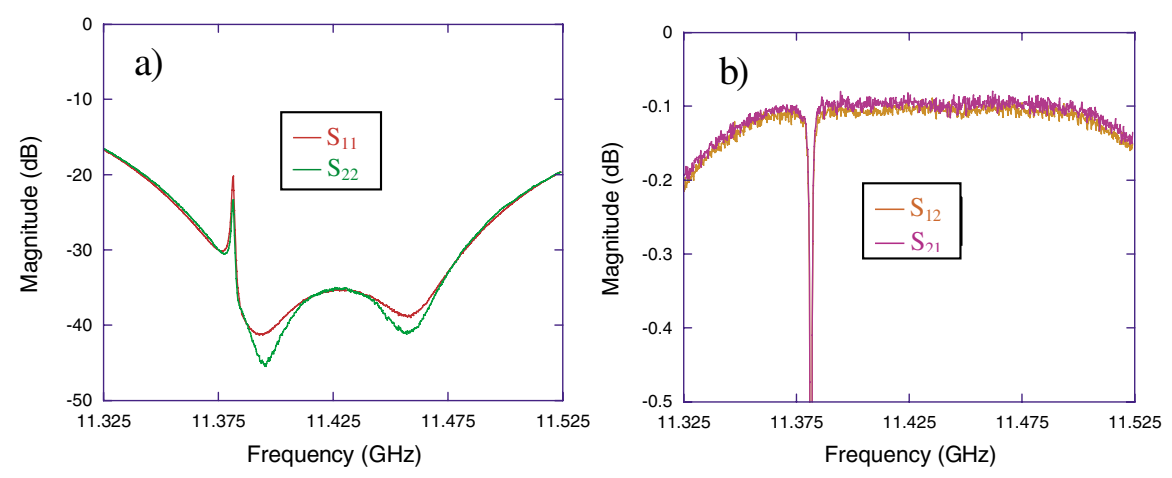

FIG. 3. (Color) Network analyzer measurements of the scattering parameters, showing (a) match and (b) transmission of two $X$-band $\mathrm{TM}_{01}$ mode converters back-to-back. 


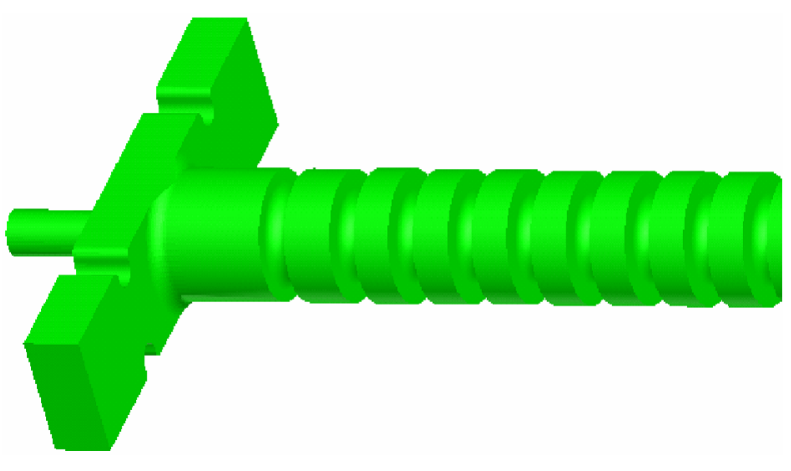

FIG. 4. (Color) Model of the $\mathrm{TM}_{01}$ mode launcher incorporated as the coupler in an accelerator structure. The first cell matches the circular waveguide to the disk-loaded structure.

acceleration is gained over the distance which it occupies. This reduces the average effective accelerating gradient of a structure. For use in a linear collider, a more compact coupler is required. With this in mind, we asked ourselves how short the circular waveguide section of a modelauncher coupler could be made before evanescent modes spoiled the independently simulated matches. It was realized that two matching steps, from rectangular waveguide to circular waveguide and from circular waveguide to disk-loaded structure, are not necessary. The $\mathrm{TM}_{01}$ waveguide can be eliminated by direct electric coupling through a circular iris in the WR90 broad wall into a matching cell. With the added design cost of having to simulate $90^{\circ}$ wedges of the $2 \mathrm{D}$ accelerator cells due to the coupler symmetry, we could, with this alternative, reduce its length by design to zero. Figure 5 illustrates the resulting waveguide coupler. The field on the input iris is low compared to that on the following irises.

This single match design also allows the elimination of bumps or posts in the rectangular waveguide ports. The WR90 is then free of obstruction. This is good from the point of view of dipole wakefield damping. One orientation of the lowest TM dipole mode excited in the adjacent structure cells by a beam excites $\mathrm{TE}_{10}$ asymmetrically in either arm of the rectangular waveguide; the other excites in-phase $\mathrm{TE}_{20}$ waves. Without matching elements, these are free to propagate out, and the modes can be

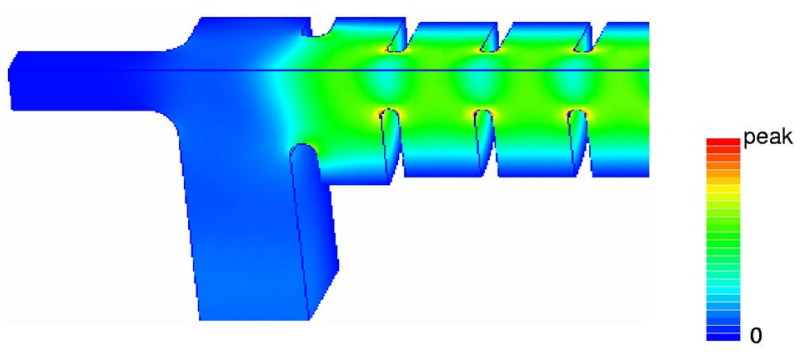

FIG. 5. (Color) Quarter geometry of a waveguide coupler with electric field plot. damped rather than trapped, as they are with the fatlipped coupler.

\section{The accelerating waveguide coupler}

Even the broad wall coupler sacrifices some acceleration efficiency for low coupler fields. There is very little field in the waveguide region, and the field in the matching cell may not be phased synchronously with the structure cells. If the couplers represent a small fraction of the structure length and/or allow a higher gradient to be reached in the main cells than otherwise achievable, it may be a viable candidate.

The nonaccelerating region can be reduced by stepping down the height of the rectangular waveguide, approaching something like a two-feed version of the ThomsonCSF coupler [8]. This, however, may lead back to unacceptably high electric and magnetic surface fields. Ideally, we would like to reclaim the coupler beam line space for acceleration. This might be accomplished with a more complicated accelerating waveguide coupler such as that shown in Fig. 6. Here partial chokes in the waveguide create a standing wave in the region of the beam line. The height of the waveguide is moderately stepped down to make it the length of a cell, and the iris coupling it to the main structure is adjusted to achieve the desired phase advance. The waveguide region thus becomes for the beam another cell. The idea is to get significant acceleration here without, in the process, raising fields at the chokes, or alternative matching elements, back to levels typical of a traditional coupler or even a fat-lipped coupler.

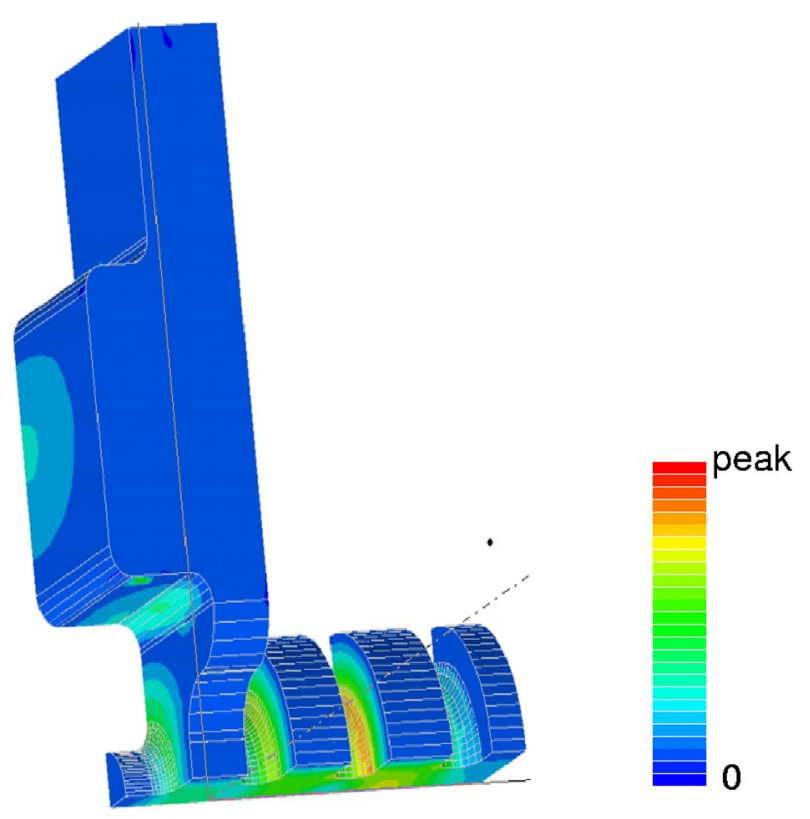

FIG. 6. (Color) Quarter geometry of an accelerating waveguide coupler with electric field plot. The fields in the waveguide region are raised to provide significant acceleration. 
The waveguide width is also stepped down near the structure axis in the design of Fig. 6, so that the half guide wavelength $\lambda_{g} / 2$ is equal to the waveguide width. This makes the lobe of the standing wave field square, eliminating quadrupole asymmetry, which can cause surface electric field enhancement on the iris and beam pipe opening. This is the equivalent of "racetracking" in the fat-lipped coupler. This conceptual coupler design has not yet been implemented in an experimental structure.

\section{MATCHING TECHNIQUES}

We now present two useful strategies for customizing couplers to particular accelerator structures with particular tables of cell dimensions. We focus on the modelauncher type, but by replacing the matching iris with their more complicated full coupler geometries and adjusting appropriate dimensions, these techniques are applicable to the other types as well.

\section{A. The symmetry method}

To determine the matching cell dimensions for a traveling-wave structure, one can use the following approach based on symmetrized models and single-mode cascading.

Neglecting evanescent modes, one can determine the characteristics required of a coupler by solving for the scattering matrices of short, symmetric structures composed of the nearest structure cells. For a constant impedance, true periodic structure, one would simply model one, two, and three cells, with waveguide ports of the cell diameter, using a mode-matching code or field solver.
Traveling-wave structures are usually designed with tapered cell dimensions to vary the group velocity and maintain a constant, or nearly constant, accelerating gradient along the structure. The upstream iris of each cell is thus different from its downstream iris, and one must artificially symmetrize a cell. To maintain true geometry adjacent to the matching plane, and because the dimensions are changing most rapidly at the ends of a structure, it is helpful to model the actual, asymmetric dimensions of the first cell or two before symmetrizing. For the next cell, the output iris is made identical to the input iris, and the outer diameter is adjusted to compensate for the effect on frequency. To first order, one can just give it the average of its original diameter and that of the preceding cell, with which it shares the iris. This cell is followed by the mirror reflection of the input cell(s). The scattering matrix at the operating frequency is determined for this geometry and again for geometries with the central, symmetric cell doubled and then tripled [see Fig. 7(a)].

If one converts such a symmetric scattering matrix $S$ into a transmission matrix $T$, cascades this between those of an undetermined matching element and its reflection as $T^{\text {tot }}=T^{m} T T^{m(1 \leftrightarrow 2)}$, and then converts back to a scattering matrix, the matching condition, $S_{11}^{\text {tot }}=0$, yields the following equation for the amplitude as a function of phase of the matching element reflection seen looking out from the structure:

$$
\left|S_{22}^{m}\right|=\frac{\cos \left(\phi_{11}+\phi_{22}^{m}\right)}{\left|S_{11}\right|}-\sqrt{\left[\frac{\cos \left(\phi_{11}+\phi_{22}^{m}\right)}{\left|S_{11}\right|}\right]^{2}-1},
$$

where the $\phi$ 's are phases of scattering matrix elements and $m$, as above, refers to the matching element matrix.

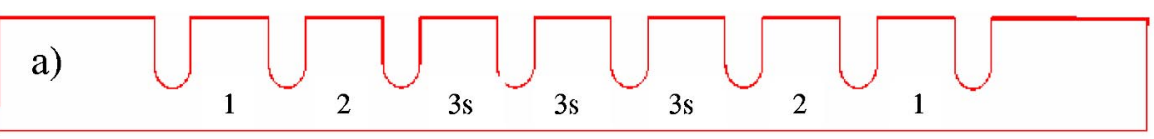

b)

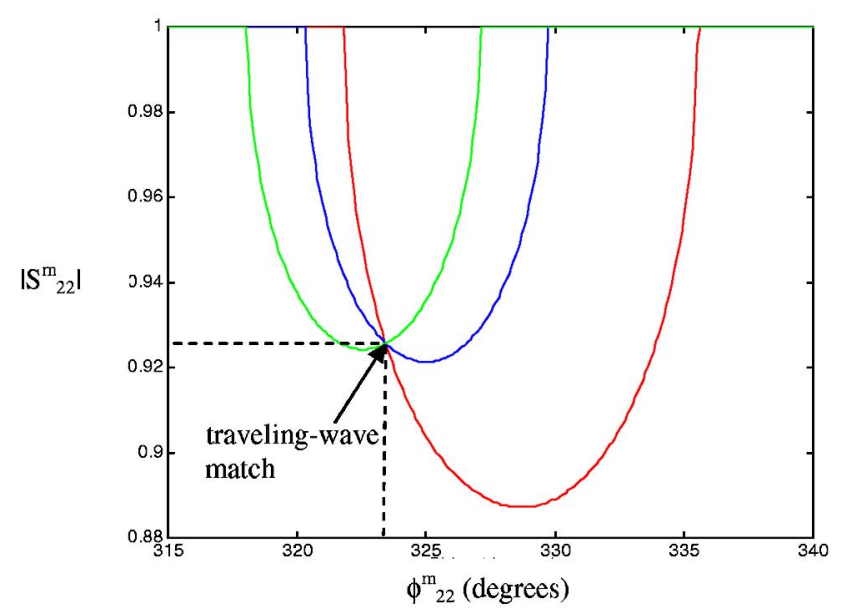

FIG. 7. (Color) (a) Profile of symmetric geometry modeled with field solver and (b) example of Eq. (1) solution for symmetric geometries composed of the initial structure cells and one (green), two (blue), and three (red) periodic central cells. 


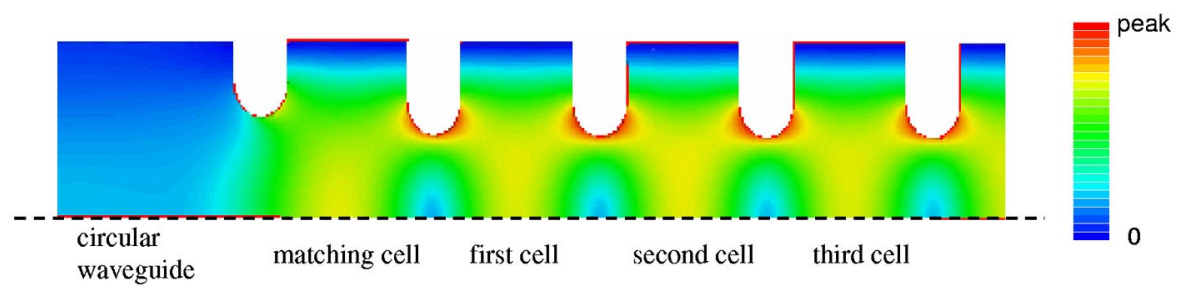

FIG. 8. (Color) Circular waveguide (left) matched to a traveling-wave periodic structure through a matching cell with electric field plot.

For each symmetric case, Eq. (1) represents a continuum of solutions for a matching complex reflection coefficient. The different cases provide different relative phases between reflections due to mismatches at either end (assuming the phase advance per cell is not $\pi$ ). Plotting the solution over the $\phi_{22}^{m}$ region where it is pure real for the cases of one, two, and three symmetrized cells, one finds that the curves intersect at a unique point, as shown in Fig. 7(b). Since this match is independent of the number of central cells, each end must be matched to a traveling wave in the periodic structure represented by those cells. Obviously, one could get away with solving only two cases to find this intersection, but doing three provides a check and allows one to reduce error by averaging the three actual intersections.

A matching iris can now be designed to give this desired reflection amplitude, and from its simulated reflection phase its proper spacing from the port, and thus from the first structure iris, can be determined, with any extra half-wavelength subtracted. This defines the matching cell. Note that with this method the diameter of the matching cell is fixed to be that of the ports of the simulated geometries. This need not be the same as the mode-converter diameter, since a diameter step can be incorporated in the design of the matching iris. For a given iris thickness the iris aperture is set to give the amplitude of the solution point, and the length of the matching cell is then set to give the phase. Figure 8 shows an example of a matched structure with a plot of the peak electric field at each point. Of course, this technique can be used to match the output end of a structure as well as the input end.

The quality of a coupling cell match can be checked by resolving the symmetrized geometries with a matching cell appended to either side. Assuming the coupler reflection is small $\left(|R|^{2} \ll 1\right)$, the complex phasor reflections from two or more such geometries, differing only in number of symmetrized cells, should lie on a circle intersecting the origin, as illustrated in Fig. 9. The center of that circle represents the reflection from a single coupler, the circle itself being the locus of points representing reflections from two couplers separated by different phase lengths. From $|a-R| \approx|b-R| \approx|R|$, with $a$ and $b$ solved reflections, we have

$$
R \approx \frac{\left(\operatorname{Im} b|a|^{2}-\operatorname{Im} a|b|^{2}\right)-i\left(\operatorname{Re} b|a|^{2}-\operatorname{Re} a|b|^{2}\right)}{2(\operatorname{Im} b \operatorname{Re} a-\operatorname{Im} a \operatorname{Re} b)}
$$

Again, a third simulation, through whose solution the circle should also pass, can provide a check. The angles between the radii connecting the center to each reflection point also provide a check on the phase advance per cell. The apparent $60^{\circ}$ angles in the figure are actually $-300^{\circ}$, reflecting a round-trip through an additional $150^{\circ}$ cell of a $5 \pi / 6$ structure.

The symmetry technique described here is applicable to more complicated three-dimensional matching problems as well, since the matching element need not be a simple waveguide iris. It can be used in designing a traditional coupler or one of the other types described in this paper. It can greatly reduce the volume that needs to be simulated as dimensions are varied by allowing the structure to be cut off from the coupler. Rather than searching directly for a match, one simply solves for the internal reflection required by the technique. Alternatively, one can solve for a simple iris match to circular waveguide, attach that coupler to one end of a

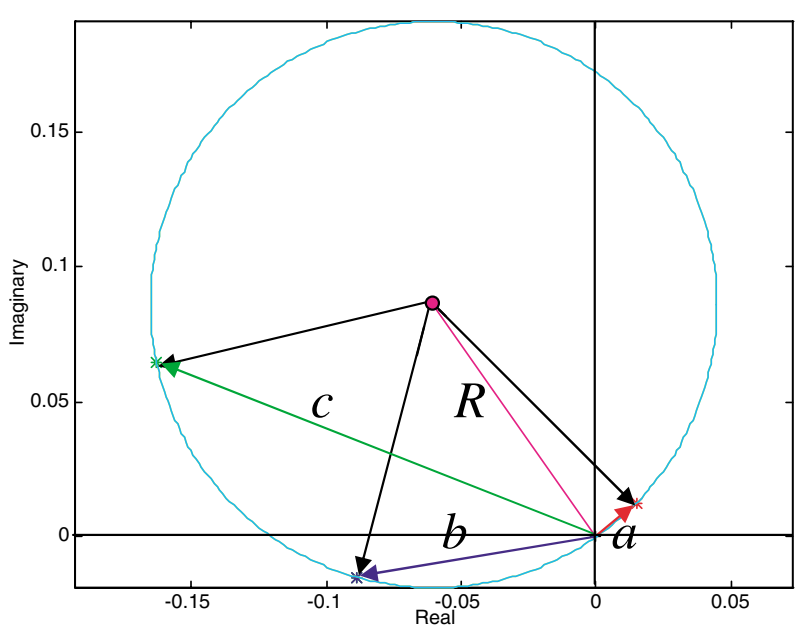

FIG. 9. (Color) Phasor plot of matching solution check. $a, b$, and $c$ are $\mathrm{S}_{11}$ values from matched symmetrized structures with three, two, and one symmetrized cells, respectively, and $R$ is the deduced residual coupler mismatch. 
short, symmetrized structure and the desired coupler type to the other end, and then vary the latter's dimensions to minimize the external reflection.

\section{B. The periodic voltage standing-wave ratio (VSWR) method}

While the symmetry method generally leads quickly to a good match, refinement of the resulting design may be required to achieve a coupler reflection much lower than $-20 \mathrm{~dB}$. The width of the traveling-wave passband affects the sensitivity, and the length of the matching cell affects the influence of evanescent higher-order modes. Coupler dimensions can be fine-tuned using a field solver and the Periodic voltage standing-wave ratio (VSWR) method as described by Kroll et al. [9]. Using formulas derived from Floquet's theorem, this method allows the internal reflection from a periodic structure coupler to be calculated from the simulated fields in the structure, with power launched toward the coupler. Taking the complex values of the on-axis electric field, $E(z-P), E(z)$, and $E(z+P)$, at three points separated by the cell period $P$, and defining

$$
\begin{aligned}
\Delta(z) & =\frac{E(z+P)-E(z-P)}{E(z)} \quad \text { and } \\
\Sigma(z) & =\frac{E(z+P)+E(z-P)}{E(z)}
\end{aligned}
$$

one can calculate the phase advance per cell $\psi$ and the reflection $R$ from the positive $z$ direction, respectively, as

$$
\psi=\cos ^{-1} \frac{\Sigma(z)}{2} \quad \text { and } \quad R=\frac{2 \sin \psi-i \Delta(z)}{2 \sin \psi+i \Delta(z)}
$$

We have used a C++ code driving the HFSS field solver to optimize coupler dimensions in an automated fashion. The optimizer moves in the two-dimensional space of the matching iris aperture diameter and either the matching cell diameter or length, modeling and solving many iterations and stepping these parameters toward a minimum of $R$, as calculated from the fields extracted through the HFSS postprocessor.

We need one frequency point calculation of a model composed of four to six cells and one coupler cell to obtain directly the coupler reflection. More than four cells are needed if the structure is tapered, as in most constant gradient accelerating structures. We note that the reflection from the excitation port is irrelevant in this method so there is no need for a second coupler in the simulation. The structure does not have to be symmetrical from beginning to end as in [9].

For typical geometries one iteration takes from 8-30 min on a two-processor $900 \mathrm{MHz}$ Pentium computer. More accurate calculations require more time. A typical coupler is matched with an overnight run of the program using lower accuracy faster iterations. Then the match is verified with several more accurate runs, which at the same time can provide tolerance analysis for manufacturing. Typical final $R$ values in the range $0.02-0.05$ are achieved.

Nine mode-launcher couplers and five fat-lipped couplers have been designed using this technique. Occasionally for output couplers, where the passband is narrow due to the lowered group velocity $(\sim 0.008 c)$, we have found it necessary to vary an additional parameter, the diameter of the first regular cell, by a few microns to perfect the match. Since optimization takes many timeconsuming field solver iterations, the two or three iterations of the symmetry technique can provide a valuable head start.

\section{CONCLUSIONS}

Recent developments in accelerator structure coupler design have been presented. The goal has been to avoid the usual field enhancement, which has been problematic in pushing to higher accelerating gradients. Of the modifications explored, a class has emerged for which the input waveguide intersects the beam line and power is coupled out through the broad wall. The methodology we have developed for coupler matching has also been treated in some detail, as a second topic.

Beyond the clear qualitative reduction of coupler surface fields to levels comparable to or below those in the structure interiors, our development has been driven by considerations such as simplicity, compactness, and acceleration, rather than further field reduction. One might expect lower fields to be achievable in a mode-launcher or waveguide coupler, which share basic matching cell geometry, than in an equivalent accelerating waveguide coupler, given the latter's on-axis field and phase advance requirement. For any coupler type, the final surface fields after matching depend on the choices one makes for fixed design parameters. For example, we have found using a thinner iris for a matching cell to lead to a solution with lower iris tip electric field. A parametric exploration of the minimal achievable surface electric or magnetic field for a given coupler type and accelerator structure is left as a subject for further study.

The mode-launcher and fat-lipped couplers were first successfully used for the input and output, respectively, of an experimental $X$-band structure, allowing it to be processed up to $90 \mathrm{MV} / \mathrm{m}$ with $400 \mathrm{~ns}$ pulses and a breakdown rate less than 0.05 per hour at a $60 \mathrm{~Hz}$ repetition rate. Several subsequent structures in the SLAC/ KEK collaboration have since been tested with modelauncher couplers to optimize the main structure parameters and fabrication techniques. Fermilab has provided an additional series of structures to the NLC R\&D program incorporating our waveguide coupler configuration [10] 
and matched with their own periodic VSWR method code. The CLIC structure program at CERN, meanwhile, has adapted our mode-converter coupler concept, with some modification [11], and also used the waveguide coupler configuration [12], for $30 \mathrm{GHz}$ test structures. While design evolution, such as that suggested by the accelerating waveguide coupler, will be needed for a final linear collider structure in order to optimize acceleration, the gradient limit imposed by coupler breakdown is now a thing of the past.

\section{ACKNOWLEDGMENTS}

This work is supported by the U.S. Department of Energy under Contract No. DE-AC03-76SF00515.

[1] G. Bowden et al., in Proceedings of the 1999 Particle Accelerator Conference, New York, 1999 (IEEE, New York, 1999), p. 3426.

[2] C. Adolphsen et al., in Proceedings of the 2001 Particle Accelerator Conference, Chicago, 2001 (IEEE, New York, 2001), p. 478.

[3] H. H. Braun et al., in Proceedings of the 21st International Linac Conference (LINAC 02), Gyeongju,
Korea, 2002 (Pohang Accelerator Laboratory, Pohang, Korea, 2003), p. 237.

[4] Valery Dolgashev, in Proceedings of the 2003 Particle Accelerator Conference (PAC 03), Portland, OR, 2003 (IEEE, New York, 2003), p. 1267.

[5] J. Haimson et al., in Proceedings of the Advanced Accelerator Concepts Workshop, Lake Tahoe, CA, 1996 (AIP, New York, 1997), p. 898.

[6] C. Adolphsen, in Proceedings of the 2003 Particle Accelerator Conference (PAC 03), Portland, OR, 2003 (Ref. [4]), p. 668; SLAC Report No. SLAC-PUB-9906.

[7] http://www.ansoft.com/products/hf/hfss/

[8] G. A. Loew and R. B. Neal, Linear Accelerators, edited by P. M. Lapostolle and A. L. Septier (North-Holland, Amsterdam, 1970), p. 97.

[9] N. M. Kroll et al., in Proceedings of the 20th International Linac Conference (LINAC 2000), Monterey, CA, 2000, edited by A. Chao (SLAC, Stanford, CA, 2000), p. 614.

[10] I. Gonin et al., in Proceedings of the 2003 Particle Accelerator Conference (PAC 03), Portland, OR, 2003 (Ref. [4]), p. 1207.

[11] I. Syratchev, CERN Report No. PS/RF/Note 2002-013, 2002.

[12] S. Doebert et al., in Proceedings of the Eighth European Particle Accelerator Conference (EPAC 02), Paris, 2002 (EPS-IGA and CERN, Geneva, 2002), p. 2187. 